\title{
Positive Solution to Nonzero Boundary Values Problem for a Coupled System of Nonlinear Fractional Differential Equations
}

\author{
Jinhua Wang, Hongjun Xiang, and Zhigang Liu \\ Department of Mathematics, Xiangnan University, Chenzhou 423000, China \\ Correspondence should be addressed to Hongjun Xiang, hunxhjxhj67@126.com
}

Received 13 April 2009; Accepted 9 June 2009

Academic Editor: Shaher Momani

Copyright (c) 2010 Jinhua Wang et al. This is an open access article distributed under the Creative Commons Attribution License, which permits unrestricted use, distribution, and reproduction in any medium, provided the original work is properly cited.

We consider the existence and uniqueness of positive solution to nonzero boundary values problem for a coupled system of fractional differential equations. The differential operator is taken in the standard Riemann-Liouville sense. By using Banach fixed point theorem and nonlinear differentiation of Leray-Schauder type, the existence and uniqueness of positive solution are obtained. Two examples are given to demonstrate the feasibility of the obtained results.

\section{Introduction}

Fractional differential equation can describe many phenomena in various fields of science and engineering such as control, porous media, electrochemistry, viscoelasticity, and electromagnetic. There are many papers dealing with the existence and uniqueness of solution for nonlinear fractional differential equation; see, for example, [1-5]. In [1], the authors investigated a singular coupled system with initial value problems of fractional order. In [2], Su discussed a boundary value problem of coupled system with zero boundary values. By means of Schauder fixed point theorem, the existence of the solution is obtained. The nonzero boundary values problem of nonlinear fractional differential equations is more difficult and complicated. No contributions exist, as far as we know, concerning the existence of positive solution for coupled system of nonlinear fractional differential equations with nonzero boundary values.

In this paper, we consider the existence and uniqueness of positive solution to nonzero boundary values problem for a coupled system of nonlinear fractional differential equations: 


$$
\begin{gathered}
D^{\alpha} u(t)+f(t, v(t))=0, \quad 0<t<1, \\
D^{\beta} v(t)+g(t, u(t))=0, \quad 0<t<1, \\
u(0)=0, \quad u(1)=a u(\xi), \\
v(0)=0, \quad v(1)=b v(\xi),
\end{gathered}
$$

where $1<\alpha<2,1<\beta<2,0 \leqslant a, b \leqslant 1,0<\xi<1, f, g:[0,1] \times[0,+\infty) \rightarrow[0,+\infty)$ are given functions, and $D$ is the standard Riemann-Liouville differentiation. By using Banach fixed point theorem and nonlinear differentiation of Leray-Schauder type, some sufficient conditions for the existence and uniqueness of positive solution to the above coupled boundary values problem are obtained.

The rest of the paper is organized as follows. In Section 2, we introduce some basic definitions and preliminaries used in later. In Section 3, the existence and uniqueness of positive solution for the coupled boundary values problem (1.1) will be discussed, and examples are given to demonstrate the feasibility of the obtained results.

\section{Basic Definitions and Preliminaries}

In this section, we introduce some basic definitions and lemmas which are used throughout this paper.

Definition 2.1 (see $[6,7])$. The fractional integral of order $\alpha(\alpha>0)$ of a function $y:(0, \infty) \rightarrow$ $R$ is given by

$$
I^{\alpha} y(t)=\frac{1}{\Gamma(\alpha)} \int_{0}^{t}(t-s)^{\alpha-1} y(s) d s
$$

provided that the right side is pointwise defined on $(0, \infty)$.

Definition 2.2 (see $[6,7]$ ). The fractional derivative of order $\alpha>0$ of a continuous function $y:(0, \infty) \rightarrow R$ is given by

$$
D^{\alpha} y(t)=\frac{1}{\Gamma(n-\alpha)}\left(\frac{d}{d t}\right)^{n} \int_{0}^{t}(t-s)^{n-\alpha-1} y(s) d s,
$$

where $n=[\alpha]+1$ provided that the right side is pointwise defined on $(0, \infty)$.

Remark 2.3 (see [3]). The following properties are useful for our discussion:

(1) $I^{\alpha} D^{\alpha} u(t)=u(t)-\sum_{k=1}^{N} C_{k} t^{\alpha-k}, D^{\alpha} u(t) \in C(0,1) \bigcap L(0,1), C_{k} \in R, N=[\alpha]+1$,

(2) $D^{\alpha} I^{\alpha} u(t)=u(t)$,

(3) $D^{\alpha} t^{\gamma}=\Gamma(\gamma+1) / \Gamma(\gamma+1-\alpha) t^{\gamma-\alpha}, \alpha>0, \gamma>-1, \gamma>\alpha-1, t>0$. 
Lemma 2.4 (the nonlinear alternative of Leray and Schauder type [8]). Let E be a Banach space with $C \subseteq E$ closed and convex. Let $U$ be a relatively open subset of $C$ with $0 \in U$ and let $T: \bar{U} \rightarrow C$ be a continuous and compact mapping. Then either

(a) the mapping $T$ has a fixed point in $\bar{U}$, or

(b) there exist $u \in \partial U$ and $\lambda \in(0,1)$ with $u=\lambda T u$.

Consider

$$
\begin{gathered}
D^{\alpha} u(t)+y(t)=0, \quad 0<t<1, \\
u(0)=0, \quad u(1)=a u(\xi),
\end{gathered}
$$

then one has the following lemma.

Lemma 2.5. Let $y \in C[0,1]$ and $1<\alpha<2$, then $u(t)$ is a solution of $B V P(2.3)$ if and only if $u(t)$ is a solution of the integral equation:

$$
u(t)=\int_{0}^{1} G_{1}(t, s) y(s) d s,
$$

where

$$
G_{1}(t, s)= \begin{cases}\frac{[t(1-s)]^{\alpha-1}-a t^{\alpha-1}(\xi-s)^{\alpha-1}-(t-s)^{\alpha-1}\left(1-a \xi^{\alpha-1}\right)}{\left(1-a \xi^{\alpha-1}\right) \Gamma(\alpha)}, & 0 \leqslant s \leqslant t \leqslant 1, s \leqslant \xi, \\ \frac{[t(1-s)]^{\alpha-1}-(t-s)^{\alpha-1}\left(1-a \xi^{\alpha-1}\right)}{\left(1-a \xi^{\alpha-1}\right) \Gamma(\alpha)}, & 0<\xi \leqslant s \leqslant t \leqslant 1, \\ \frac{[t(1-s)]^{\alpha-1}-a t^{\alpha-1}(\xi-s)^{\alpha-1}}{\left(1-a \xi^{\alpha-1}\right) \Gamma(\alpha)}, & 0 \leqslant t \leqslant s \leqslant \xi \leqslant 1, \\ \frac{[t(1-s)]^{\alpha-1}}{\left(1-a \xi^{\alpha-1}\right) \Gamma(\alpha)}, & 0 \leqslant t \leqslant s \leqslant 1, \xi \leqslant s .\end{cases}
$$

Proof. Assume that $u(t)$ is a solution of BVP (2.3), then by Remark 2.3, we have

$$
\begin{aligned}
u(t) & =-I^{\alpha} y(t)+C_{1} t^{\alpha-1}+C_{2} t^{\alpha-2} \\
& =-\int_{0}^{t} \frac{(t-s)^{\alpha-1}}{\Gamma(\alpha)} y(s) d s+C_{1} t^{\alpha-1}+C_{2} t^{\alpha-2} .
\end{aligned}
$$

By (2.3), we have

$$
C_{2}=0, \quad C_{1}=\int_{0}^{1} \frac{(1-s)^{\alpha-1}}{\Gamma(\alpha)\left(1-a \xi^{\alpha-1}\right)} y(s) d s-a \int_{0}^{\xi} \frac{(\xi-s)^{\alpha-1}}{\Gamma(\alpha)\left(1-a \xi^{\alpha-1}\right)} y(s) d s .
$$


Therefore, we obtain

$$
\begin{aligned}
u(t) & =-\int_{0}^{t} \frac{(t-s)^{\alpha-1}}{\Gamma(\alpha)} y(s) d s+\int_{0}^{1} \frac{t^{\alpha-1}(1-s)^{\alpha-1}}{\Gamma(\alpha)\left(1-a \xi^{\alpha-1}\right)} y(s) d s-a \int_{0}^{\xi} \frac{t^{\alpha-1}(\xi-s)^{\alpha-1}}{\Gamma(\alpha)\left(1-a \xi^{\alpha-1}\right)} y(s) d s \\
& =\int_{0}^{1} G_{1}(t, s) y(s) d s .
\end{aligned}
$$

Conversely, if $u(t)$ is a solution of integral equation (2.4), using the relation $D^{\alpha} t^{\alpha-m}=$ $0, m=1,2, \ldots, N$, where $N$ is the smallest integer greater than or equal to $\alpha$ [3, Remark 2.1], we have

$$
\begin{aligned}
D^{\alpha} u(t)= & -D^{\alpha}\left(\int_{0}^{t} \frac{(t-s)^{\alpha-1}}{\Gamma(\alpha)} y(s) d s\right) \\
& +D^{\alpha} t^{\alpha-1}\left[\int_{0}^{1} \frac{(1-s)^{\alpha-1}}{\Gamma(\alpha)\left(1-a \xi^{\alpha-1}\right)} y(s) d s-a \int_{0}^{\xi} \frac{(\xi-s)^{\alpha-1}}{\Gamma(\alpha)\left(1-a \xi^{\alpha-1}\right)} y(s) d s\right] \\
= & -D^{\alpha} I^{\alpha} y(t)=-y(t) .
\end{aligned}
$$

A simple computation showed $u(0)=0, u(1)=a u(\xi)$. The proof is complete.

Let

$$
G_{2}(t, s)= \begin{cases}\frac{[t(1-s)]^{\beta-1}-b t^{\beta-1}(\xi-s)^{\beta-1}-(t-s)^{\beta-1}\left(1-b \xi^{\beta-1}\right)}{\left(1-b \xi^{\beta-1}\right) \Gamma(\beta)}, & 0 \leqslant s \leqslant t \leqslant 1, s \leqslant \xi, \\ \frac{[t(1-s)]^{\beta-1}-(t-s)^{\beta-1}\left(1-b \xi^{\beta-1}\right)}{\left(1-b \xi^{\beta-1}\right) \Gamma(\beta)}, & 0<\xi \leqslant s \leqslant t \leqslant 1, \\ \frac{[t(1-s)]^{\beta-1}-b t^{\beta-1}(\xi-s)^{\beta-1}}{\left(1-b \xi^{\beta-1}\right) \Gamma(\beta)}, & 0 \leqslant t \leqslant s \leqslant \xi \leqslant 1, \\ \frac{[t(1-s)]^{\beta-1}}{\left(1-b \xi^{\beta-1}\right) \Gamma(\beta)}, & 0 \leqslant t \leqslant s \leqslant 1, \xi \leqslant s,\end{cases}
$$

we call $G(t, s)=\left(G_{1}(t, s), G_{2}(t, s)\right)$ Green's function of the boundary value problem (1.1).

Lemma 2.6. Let $0 \leqslant a, b \leqslant 1$, then the function $G(t, s)$ is continuous and satisfies

(1) $G(t, s)>0$, for $t, s \in(0,1)$,

(2) $G(t, s) \leqslant G(s, s)$, for $t, s \in(0,1)$. 
Proof. It is easy to prove that $G(t, s)$ is continuous on $[0,1] \times[0,1]$, here we omit it. Now we prove $G_{1}(t, s)>0$. Let

$$
\begin{aligned}
& g_{1}(t, s)=\frac{[t(1-s)]^{\alpha-1}-a t^{\alpha-1}(\xi-s)^{\alpha-1}-(t-s)^{\alpha-1}\left(1-a \xi^{\alpha-1}\right)}{\left(1-a \xi^{\alpha-1}\right) \Gamma(\alpha)}, \quad 0<s \leqslant t \leqslant 1, s \leqslant \xi, \\
& g_{2}(t, s)=\frac{[t(1-s)]^{\alpha-1}-(t-s)^{\alpha-1}\left(1-a \xi^{\alpha-1}\right)}{\left(1-a \xi^{\alpha-1}\right) \Gamma(\alpha)}, \quad 0<\xi \leqslant s \leqslant t \leqslant 1, \\
& g_{3}(t, s)=\frac{[t(1-s)]^{\alpha-1}-a t^{\alpha-1}(\xi-s)^{\alpha-1}}{\left(1-a \xi^{\alpha-1}\right) \Gamma(\alpha)}, \quad 0<t \leqslant s \leqslant \xi \leqslant 1, \\
& g_{4}(t, s)=\frac{[t(1-s)]^{\alpha-1}}{\left(1-a \xi^{\alpha-1}\right) \Gamma(\alpha)}, \quad 0<t \leqslant s \leqslant 1, \xi \leqslant s .
\end{aligned}
$$

We only need to prove $g_{1}(t, s)>0,0<s \leqslant t \leqslant 1, s \leqslant \xi$. Since

$$
\begin{aligned}
& {[t(1-s)]^{\alpha-1}-a t^{\alpha-1}(\xi-s)^{\alpha-1}-(t-s)^{\alpha-1}\left(1-a \xi^{\alpha-1}\right)} \\
& \quad=t^{\alpha-1}[(1-s)]^{\alpha-1}-a(\xi-s)^{\alpha-1}-\left(1-\frac{s}{t}\right)^{\alpha-1}\left(1-a \xi^{\alpha-1}\right),
\end{aligned}
$$

set $g(t)=(1-s)^{\alpha-1}-a(\xi-s)^{\alpha-1}-(1-s / t)^{\alpha-1}\left(1-a \xi^{\alpha-1}\right)$, we have

$$
g^{\prime}(t)=-(\alpha-1)\left(1-\frac{s}{t}\right)^{\alpha-2} \frac{s}{t^{2}}\left(1-a \xi^{\alpha-1}\right) \leqslant 0, \quad \text { for } 0<s<t \leqslant 1, s \leqslant \xi .
$$

Then $g(t)$ is decreasing on $(0,1)$. Meanwhile,

$$
\begin{aligned}
g(1) & =(1-s)^{\alpha-1}-a(\xi-s)^{\alpha-1}-(1-s)^{\alpha-1}\left(1-a \xi^{\alpha-1}\right) \\
& =a \xi^{\alpha-1}\left[(1-s)^{\alpha-1}-\left(1-\frac{s}{\xi}\right)^{\alpha-1}\right]>0, \quad 0<s<t \leqslant 1, s \leqslant \xi .
\end{aligned}
$$

Therefore, $g_{1}(t, s)>0$, for $0<s<t \leqslant 1, s \leqslant \xi$. Clearly $g_{1}(t, s)>0, t=s$, so $g_{1}(t, s)>$ $0, s, t \in(0,1)$. It is easy to show that $g_{2}(t, s)>0, g_{3}(t, s)>0, g_{4}(t, s)>0$. Hence, $G_{1}(t, s)>$ $0, s, t \in(0,1)$.

Similarly, $G_{2}(t, s)>0, s, t \in(0,1)$. The proof of (1) is completed.

Let

$$
g_{2}(t)=\frac{[t(1-s)]^{\alpha-1}-(t-s)^{\alpha-1}\left(1-a \xi^{\alpha-1}\right)}{\left(1-a \xi^{\alpha-1}\right) \Gamma(\alpha)}, \quad 0<\xi \leqslant s \leqslant t \leqslant 1,
$$


then,

$$
\begin{gathered}
g_{2}^{\prime}(t)=\frac{(\alpha-1) t^{\alpha-2}[(1-s)]^{\alpha-1}-(1-s / t)^{\alpha-2}\left(1-a \xi^{\alpha-1}\right)}{\left(1-a \xi^{\alpha-1}\right) \Gamma(\alpha)}, 0<\xi \leqslant s<t \leqslant 1, \\
{[(1-s)]^{\alpha-1}-(1-s / t)^{\alpha-2}\left(1-a \xi^{\alpha-1}\right)} \\
\leqslant[(1-s)]^{\alpha-1}-(1-s)^{\alpha-2}\left(1-a \xi^{\alpha-1}\right) \\
=[(1-s)]^{\alpha-2}\left(a \xi^{\alpha-1}-s\right) \leqslant 0, \quad 0<\xi \leqslant s<t \leqslant 1,
\end{gathered}
$$

therefore,

$$
g_{2}^{\prime}(t) \leqslant 0, \quad 0<\xi \leqslant s<t \leqslant 1
$$

So, $g_{2}(t, s)$ is decreasing with respect to $t$. Similarly, $g_{1}(t, s)$ is decreasing with respect to $t$. Also $g_{3}(t, s)$ and $g_{4}(t, s)$ are increasing with respect to $t$. We obtain that $G_{1}(t, s)$ is decreasing with respect to $t$ for $s \leqslant t$ and increasing with respect to $t$ for $t \leqslant s$.

With the use of the monotonicity of $G_{1}(t, s)$, we have

$$
\max _{0 \leqslant t \leqslant 1} G_{1}(t, s)=G_{1}(s, s)= \begin{cases}\frac{[s(1-s)]^{\alpha-1}-a[s(\xi-s)]^{\alpha-1}}{\Gamma(\alpha)\left(1-a \xi^{\alpha-1}\right)}, & s \in(0, \xi], \\ \frac{[s(1-s)]^{\alpha-1}}{\Gamma(\alpha)\left(1-a \xi^{\alpha-1}\right)}, & s \in[\xi, 1) .\end{cases}
$$

Similarly,

$$
\max _{0 \leqslant t \leqslant 1} G_{2}(t, s)=G_{2}(s, s)= \begin{cases}\frac{[s(1-s)]^{\beta-1}-b[s(\xi-s)]^{\beta-1}}{\Gamma(\beta)\left(1-b \xi^{\beta-1}\right)}, & s \in(0, \xi], \\ \frac{[s(1-s)]^{\beta-1}}{\Gamma(\beta)\left(1-b \xi^{\beta-1}\right)}, & s \in[\xi, 1) .\end{cases}
$$

The proof of (2) is completed.

\section{Main Result}

In this section, we will discuss the existence and uniqueness of positive solution for boundary value problem (1.1).

We define the space $X=\{u(t) \mid u(t) \in C[0,1]\}$ endowed with $\|u\|_{X}=\max _{0 \leqslant t \leqslant 1}|u(t)|$, $Y=\{v(t) \mid v(t) \in C[0,1]\}$ endowed with $\|u\|_{Y}=\max _{0 \leqslant t \leqslant 1}|v(t)|$.

For $(u, v) \in X \times Y$, let $\|(u, v)\|_{X \times Y}=\max \left\{\|u\|_{X},\|v\|_{Y}\right\}$.

Define $P=\{(u, v) \in X \times Y \mid u(t) \geqslant 0, v(t) \geqslant 0\}$, then the cone $P \subset X \times Y$. 
From Lemma 2.5 in Section 2, we can obtain the following lemma.

Lemma 3.1. Suppose that $f(t, v)$ and $g(t, u)$ are continuous, then $(u, v) \in X \times Y$ is a solution of $B V P(1.1)$ if and only if $(u, v) \in X \times Y$ is a solution of the integral equations

$$
\begin{aligned}
& u(t)=\int_{0}^{1} G_{1}(t, s) f(s, v(s)) d s, \\
& v(t)=\int_{0}^{1} G_{2}(t, s) g(s, u(s)) d s .
\end{aligned}
$$

Let $T: X \times Y \rightarrow X \times Y$ be the operator defined as

$$
\begin{aligned}
T(u, v)(t) & =\left(\int_{0}^{1} G_{1}(t, s) f(s, v(s)) d s, \int_{0}^{1} G_{2}(t, s) g(s, u(s)) d s\right) \\
& =:\left(T_{1} v(t), T_{2} u(t)\right),
\end{aligned}
$$

then by Lemma 3.1, the fixed point of operator $T$ coincides with the solution of system (1.1).

Lemma 3.2. Let $f(t, v)$ and $g(t, u)$ be continuous on $[0,1] \times[0, \infty) \rightarrow[0, \infty)$, then $T: P \rightarrow P$ defined by (3.2) is completely continuous.

Proof. Let $(u, v) \in P$, in view of nonnegativeness and continuity of functions $G(t, s), f$, and $g$, we conclude that $T: P \rightarrow P$ is continuous.

Let $\Omega \in P$ be bounded, that is, there exists a positive constant $h>0$ such that $\|(u, v)\| \leqslant$ $h$ for all $(u, v) \in \Omega$.

Let

$$
\begin{aligned}
& M=\max \{|f(t, v(t))|+1: 0 \leqslant t \leqslant 1,0 \leqslant v \leqslant h\}, \\
& N=\max \{|g(t, u(t))|+1: 0 \leqslant t \leqslant 1,0 \leqslant u \leqslant h\},
\end{aligned}
$$

then we have

$$
\begin{aligned}
& \left|T_{1} v(t)\right|=\left|\int_{0}^{1} G_{1}(t, s) f(s, v(s)) d s\right| \leqslant M \int_{0}^{1} G_{1}(s, s) d s, \\
& \left|T_{2} u(t)\right|=\left|\int_{0}^{1} G_{2}(t, s) g(s, u(s)) d s\right| \leqslant N \int_{0}^{1} G_{2}(s, s) d s .
\end{aligned}
$$

Hence, $\|T(u, v)\| \leqslant \max \left\{M \int_{0}^{1} G_{1}(s, s) d s, N \int_{0}^{1} G_{2}(s, s) d s\right\}$. $T(\Omega)$ is uniformly bounded.

Since $G_{1}(t, s)$ is continuous on $[0,1] \times[0,1]$, it is uniformly continuous on $[0,1] \times[0,1]$. Thus, for fixed $s \in[0,1]$ and for any $\varepsilon>0$, there exists a constant $\delta>0$, such that any $t_{1}, t_{2} \in[0,1]$ and $\left|t_{1}-t_{2}\right|<\delta$,

$$
\left|G_{1}\left(t_{1}, s\right)-G_{1}\left(t_{2}, s\right)\right|<\varepsilon / M
$$


Then

$$
\left|T_{1}(v)\left(t_{2}\right)-T_{1}(v)\left(t_{1}\right)\right| \leqslant M \int_{0}^{1}\left|G_{1}\left(t_{2}, s\right)-G_{1}\left(t_{1}, s\right)\right| d s<\varepsilon
$$

Similarly,

$$
\left|T_{2}(u)\left(t_{2}\right)-T_{2}(u)\left(t_{1}\right)\right| \leqslant N \int_{0}^{1}\left|G_{2}\left(t_{2}, s\right)-G_{2}\left(t_{1}, s\right)\right| d s<\varepsilon .
$$

For the Euclidean distance $d$ on $R^{2}$, we have that if $t_{1}, t_{2} \in[0,1]$ are such that $\left|t_{2}-t_{1}\right|<\delta$, then

$$
d\left(T(u, v)\left(t_{2}\right), T(u, v)\left(t_{1}\right)\right)=\sqrt{\left(T_{1} v\left(t_{2}\right)-T_{1} v\left(t_{1}\right)\right)^{2}+\left(T_{2} u\left(t_{2}\right)-T_{2} u\left(t_{1}\right)\right)^{2}}<\sqrt{2} \varepsilon
$$

That is to say, $T(P)$ is equicontinuous. By the means of the Arzela-Ascoli theorem, we have $T: P \rightarrow P$ is completely continuous. The proof is completed.

Theorem 3.3. Assume that $f(t, v)$ and $g(t, u)$ are continuous on $[0,1] \times[0, \infty) \rightarrow[0, \infty)$, and there exist two positive functions $m(t), n(t)$ that satisfy

$$
\begin{aligned}
& \left(H_{1}\right)\left|f\left(t, v_{2}\right)-f\left(t, v_{1}\right)\right| \leqslant m(t)\left|v_{2}-v_{1}\right|, \text { for } t \in[0,1], v_{1}, v_{2} \in[0, \infty), \\
& \left(H_{2}\right)\left|g\left(t, u_{2}\right)-g\left(t, u_{1}\right)\right| \leqslant n(t)\left|u_{2}-u_{1}\right|, \text { for } t \in[0,1], u_{1}, u_{2} \in[0, \infty) .
\end{aligned}
$$

Then system (1.1) has a unique positive solution if

$$
\rho=\int_{0}^{1} G_{1}(s, s) m(s) d s<1, \quad \theta=\int_{0}^{1} G_{2}(s, s) n(s) d s<1 .
$$

Proof. For all $(u, v) \in P$, by the nonegativeness of $G(t, s)$ and $f(t, v), g(t, u)$, we have $T(u, v)(t) \geqslant 0$. Hence, $T(P) \subseteq P$.

$$
\begin{aligned}
\left\|T_{1} v_{2}-T_{1} v_{1}\right\| & =\max _{t \in[0,1]}\left|T_{1} v_{2}-T_{1} v_{1}\right| \\
& =\max _{t \in[0,1]}\left|\int_{0}^{1} G_{1}(t, s)\left[f\left(s, v_{2}(s)\right)-f\left(s, v_{1}(s)\right)\right] d s\right| \\
& \leqslant \int_{0}^{1} G_{1}(s, s) m(s) d s\left\|v_{2}-v_{1}\right\| \\
& \leqslant \rho\left\|v_{2}-v_{1}\right\| .
\end{aligned}
$$

Similarly,

$$
\left\|T_{2} u_{2}-T_{2} u_{1}\right\| \leqslant \theta\left\|u_{2}-u_{1}\right\|
$$


We have,

$$
\left\|T\left(u_{2}, v_{2}\right)-T\left(u_{1}, v_{1}\right)\right\| \leqslant \max (\rho, \theta)\left\|\left(u_{2}, v_{2}\right)-\left(u_{1}, v_{1}\right)\right\| .
$$

From Lemma 3.2, $T$ is completely continuous, by Banach fixed point theorem, the operator $T$ has a unique fixed point in $P$, which is the unique positive solution of system (1.1). This completes the proof.

Theorem 3.4. Assume that $f(t, v)$ and $g(t, u)$ are continuous on $[0,1] \times[0, \infty) \rightarrow[0, \infty)$ and satisfy

$\left(H_{3}\right)|f(t, v(t))| \leqslant a_{1}(t)+a_{2}(t)|v(t)|$,

$\left(H_{4}\right)|g(t, u(t))| \leqslant b_{1}(t)+b_{2}(t)|u(t)|$,

$\left(H_{5}\right) A_{1}=\int_{0}^{1} G_{1}(s, s) a_{2}(s) d s<1,0<B_{1}=\int_{0}^{1} G_{1}(s, s) a_{1}(s) d s<\infty$,

$\left(H_{6}\right) A_{2}=\int_{0}^{1} G_{2}(s, s) b_{2}(s) d s<1,0<B_{2}=\int_{0}^{1} G_{2}(s, s) b_{1}(s) d s<\infty$.

Then the system (1.1) has at least one positive solution $(u, v)$ in

$$
C=\left\{(u, v) \in P \mid\|(u, v)\|<\min \left(\frac{B_{1}}{1-A_{1}}, \frac{B_{2}}{1-A_{2}}\right)\right\} .
$$

Proof. Let $C=\{(u, v) \in X \times Y:\|(u, v)\|<r\}$ with $r=\min \left(B_{1} /\left(1-A_{1}\right), B_{2} /\left(1-A_{2}\right)\right)$, define the operator $T: C \rightarrow P$ as (3.2).

Let $(u, v) \in C$, that is, $\|(u, v)\|<r$. Then

$$
\begin{aligned}
\left\|T_{1} v\right\| & =\max _{t \in[0,1]}\left|\int_{0}^{1} G_{1}(t, s) f(s, v(s)) d s\right| \\
& \leqslant \int_{0}^{1} G_{1}(s, s)\left(a_{1}(s)+a_{2}(s)|v(s)|\right) d s \\
& \leq \int_{0}^{1} G_{1}(s, s) a_{1}(s) d s+\int_{0}^{1} G_{1}(s, s) a_{2}(s) d s\|v\| \\
& =B_{1}+A_{1}\|v\| \leqslant r .
\end{aligned}
$$

Similarly, $\left\|T_{2} u\right\| \leqslant r$, so $\|T(u, v)\| \leqslant r, T(u, v) \subseteq \bar{C}$. From Lemma 3.2 $T: \bar{C} \rightarrow \bar{C}$ is completely continuous.

Consider the eigenvalue problem

$$
(u, v)=\lambda T(u, v), \quad \lambda \in(0,1) .
$$


Under the assumption that $(u, v)$ is a solution of $(3.15)$ for a $\lambda \in(0,1)$, one obtains

$$
\begin{aligned}
\|u\| & =\left\|\lambda T_{1} v\right\| \\
& =\lambda \max _{t \in[0,1]}\left|\int_{0}^{1} G_{1}(t, s) f(s, v(s)) d s\right| \\
& <\int_{0}^{1} G_{1}(s, s)\left(a_{1}(s)+a_{2}(s)|v(s)|\right) d s \\
& =\int_{0}^{1} G_{1}(s, s) a_{1}(s) d s+\int_{0}^{1} G_{1}(s, s) a_{2}(s) d s\|v\| \\
& =B_{1}+A_{1}\|v\| \leqslant r .
\end{aligned}
$$

Similarly, $\|v\|=\left\|\lambda T_{2} u\right\|<r$, so $\|(u, v)\|<r$, which shows that $(u, v) \notin \partial C$. By Lemma 2.4, $T$ has a fixed point in $\bar{C}$. We complete the proof of Theorem 3.4.

Example 3.5. Consider the problem

$$
\begin{gathered}
D^{7 / 4} u(t)+f(t, v(t))=0, \quad 0<t<1, \\
D^{3 / 2} v(t)+g(t, u(t))=0, \quad 0<t<1, \\
u(0)=0, \quad u(1)=\frac{1}{2} u\left(\frac{1}{2}\right), \\
v(0)=0, \quad v(1)=\frac{3}{4} v\left(\frac{1}{2}\right),
\end{gathered}
$$

where

$$
f(t, v(t))=\frac{t v(t)}{(1+t)(1+v(t))}, \quad g(t, u(t))=\arctan \frac{t}{1+t}|\sin u(t)|
$$

Set $v_{1}(t), v_{2}(t), u_{1}(t), u_{2}(t) \in[0, \infty)$ and $t \in[0,1]$, then we have

$$
\begin{aligned}
& \left|f\left(t, v_{2}(t)\right)-f\left(t, v_{1}(t)\right)\right| \leqslant \frac{t}{1+t}\left|v_{2}(t)-v_{1}(t)\right|, \\
& \left|g\left(t, u_{2}(t)\right)-g\left(t, u_{1}(t)\right)\right| \leqslant \arctan \frac{t}{1+t}\left|u_{2}(t)-u_{1}(t)\right| .
\end{aligned}
$$


Therefore,

$$
\begin{aligned}
\rho & =\int_{0}^{1} G_{1}(s, s) m(s) d s \leqslant \int_{0}^{1} G_{1}(s, s) d s \\
& =\frac{1}{\Gamma(7 / 4)\left(1-(1 / 2)^{7 / 4}\right)}\left\{\int_{0}^{1 / 2}[s(1-s)]^{3 / 4} d s-\int_{0}^{1 / 2} \frac{1}{2}\left[s\left(\frac{1}{2}-s\right)\right]^{3 / 4} d s+\int_{1 / 2}^{1}[s(1-s)]^{3 / 4} d s\right\} \\
& =\frac{2\left(1+(1 / 2)^{7 / 4}\right)}{5} \cdot \frac{\Gamma(3 / 4)}{\Gamma(1 / 2)}<\frac{4}{5}<1, \\
\theta & =\int_{0}^{1} G_{2}(s, s) n(s) d s \leqslant \frac{\pi}{4} \int_{0}^{1} G_{2}(s, s) d s \\
& =\frac{\pi}{4} \frac{1}{\Gamma(3 / 2)\left(1-(3 / 4)(1 / 2)^{1 / 2}\right)}\left\{\int_{0}^{1 / 2}[s(1-s)]^{1 / 2} d s-\int_{0}^{1 / 2} \frac{3}{4}\left[s\left(\frac{1}{2}-s\right)\right]^{1 / 2} d s+\int_{1 / 2}^{1}[s(1-s)]^{1 / 2} d s\right\} \\
& =\frac{\pi}{4} \frac{\left[1-(3 / 4)(1 / 2)^{2}\right]}{\left[1-(3 / 4)(1 / 2)^{1 / 2}\right]} \cdot \frac{\Gamma(3 / 2)}{\Gamma(3)} \\
& \approx 0.6018<1 .
\end{aligned}
$$

With the use of Theorem 3.3, BVP (3.17) has a unique positive solution.

Example 3.6. Consider the problem

$$
\begin{gathered}
D^{7 / 4} u(t)+f(t, v(t))=0, \quad 0<t<1, \\
D^{3 / 2} v(t)+g(t, u(t))=0, \quad 0<t<1, \\
u(0)=0, \quad u(1)=\frac{1}{2} u\left(\frac{1}{2}\right), \\
v(0)=0, \quad v(1)=\frac{3}{4} v\left(\frac{1}{2}\right),
\end{gathered}
$$

where

$$
f(t, v(t))=t^{2}+\frac{t}{1+t} \ln (1+v(t)), \quad g(t, u(t))=10+\frac{t^{2}}{20}+u(t) .
$$

We have

$$
|f(t, v(t))| t^{2}+\frac{t}{1+t} \cdot|v(t)|, \quad|g(t, u(t))| \leqslant\left(10+\frac{t^{2}}{20}\right)+|u(t)|
$$


Hence,

$$
\begin{aligned}
& A_{1}=\int_{0}^{1} G_{1}(s, s) a_{2}(s) d s \leqslant \int_{0}^{1} G_{1}(s, s) d s=\frac{2\left(1+(1 / 2)^{7 / 4}\right)}{5} \cdot \frac{\Gamma(3 / 4)}{\Gamma(1 / 2)}<1, \\
& B_{1}=\int_{0}^{1} G_{1}(s, s) a_{1}(s) d s=\int_{0}^{1} G_{1}(s, s) \cdot s^{2} d s<\infty, \\
& A_{2}=\int_{0}^{1} G_{2}(s, s) b_{2}(s) d s=\int_{0}^{1} G_{2}(s, s) d s \approx 0.7666<1, \\
& B_{2}=\int_{0}^{1} G_{2}(s, s) b_{1}(s) d s=\int_{0}^{1} G_{2}(s, s)\left(10+\frac{s^{2}}{20}\right) d s<\infty .
\end{aligned}
$$

By Theorem 3.4, BVP (3.21) has at least one positive solution in

$$
C=\left\{(u, v) \in P \mid\|u, v\|<\min \left(\frac{B_{1}}{1-A_{1}}, \frac{B_{2}}{1-A_{2}}\right)\right\}
$$

\section{Acknowledgments}

This work was jointly supported by the Natural Science Foundation of Hunan Provincial Education Department under Grants 07A066 and 07C700, the Construct Program of the Key Discipline in Hunan Province, Aid Program for Science and Technology Innovative Research Team in Higher Educational Institutions of Hunan Province, and the Foundation of Xiangnan University.

\section{References}

[1] C. Bai and J. Fang, "The existence of a positive solution for a singular coupled system of nonlinear fractional differential equations," Applied Mathematics and Computation, vol. 150, no. 3, pp. 611-621, 2004.

[2] X. Su, "Boundary value problem for a coupled system of nonlinear fractional differential equations," Applied Mathematics Letters, vol. 22, no. 1, pp. 64-69, 2009.

[3] Z. Bai and H. Lü, "Positive solutions for boundary value problem of nonlinear fractional differential equation," Journal of Mathematical Analysis and Applications, vol. 311, no. 2, pp. 495-505, 2005.

[4] S. Zhang, "Existence of solution for a boundary value problem of fractional order," Acta Mathematica Scientia, vol. 26, no. 2, pp. 220-228, 2006.

[5] S. Q. Zhang, "Positive of solution for boundary-value problems of nonlinear fractional differential equations," Electronic Journal of Differential Equations, vol. 36, pp. 1-12, 2006.

[6] I. Podlubny, Fractional Differential Equations, vol. 198 of Mathematics in Science and Engineering, Academic Press, San Diego, Calif, USA, 1999.

[7] A. A. Kilbas, H. M. Srivastava, and J. J. Trujillo, Theory and Applications of Fractional Differential Equations, vol. 204 of North-Holland Mathematics Studies, Elsevier Science, Amsterdam, The Netherlands, 2006.

[8] E. Zeidler, Nonlinear Functional Analysis and Its Applications-I: Fixed-Point Theorems, Springer, New York, NY, USA, 1986. 


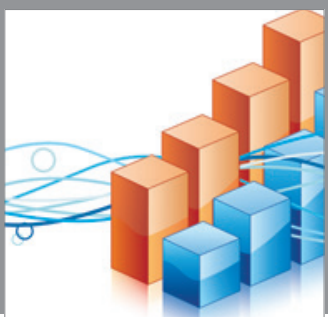

Advances in

Operations Research

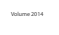

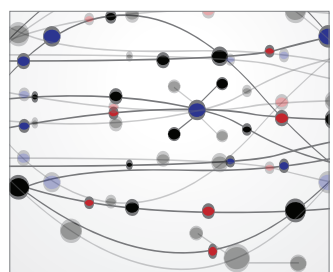

\section{The Scientific} World Journal
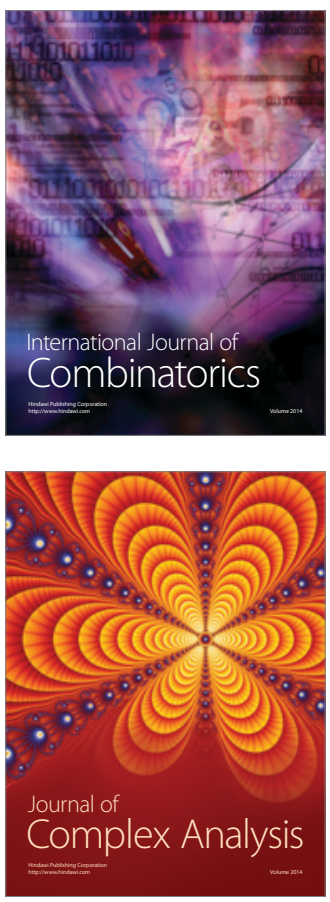

International Journal of

Mathematics and

Mathematical

Sciences
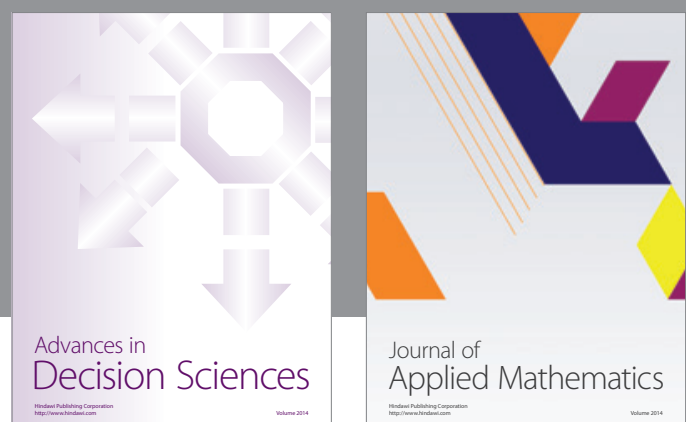

Journal of

Applied Mathematics
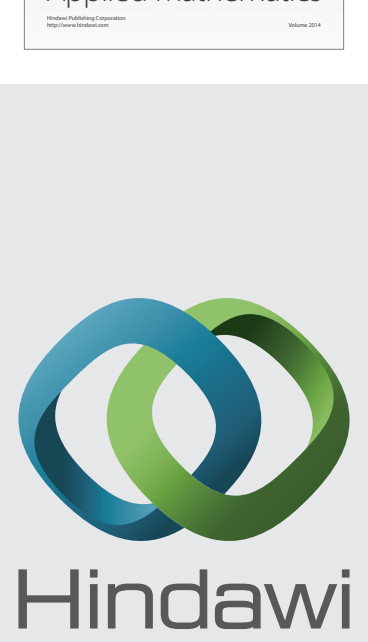

Submit your manuscripts at http://www.hindawi.com
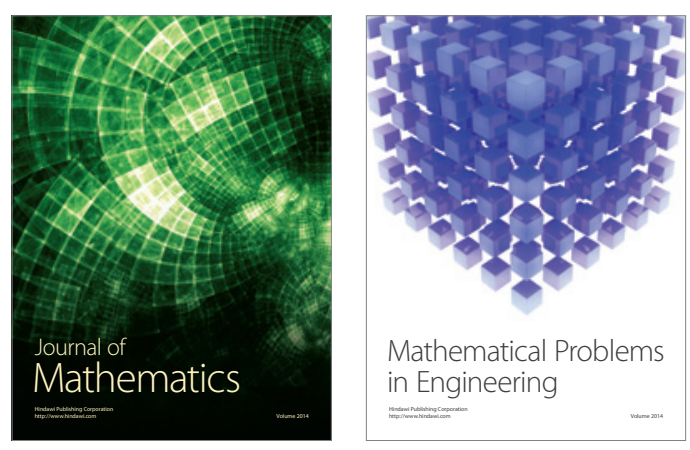

Mathematical Problems in Engineering
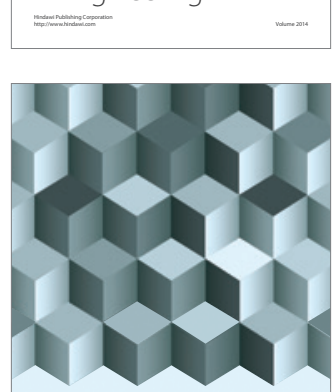

Journal of

Function Spaces
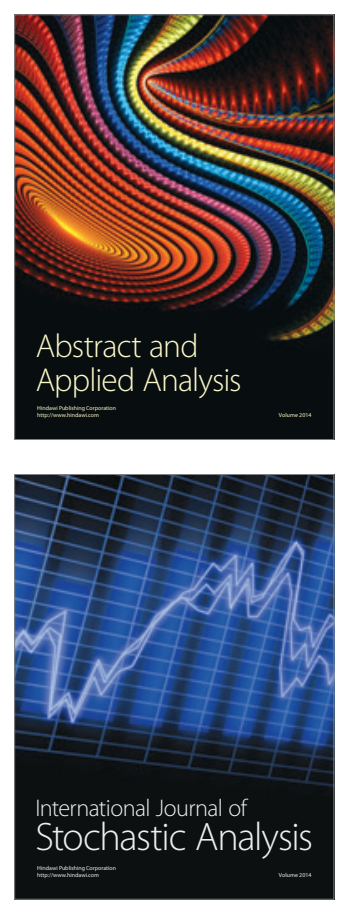

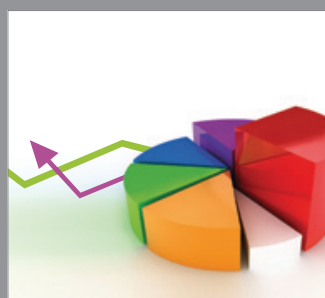

ournal of

Probability and Statistics

Promensencen
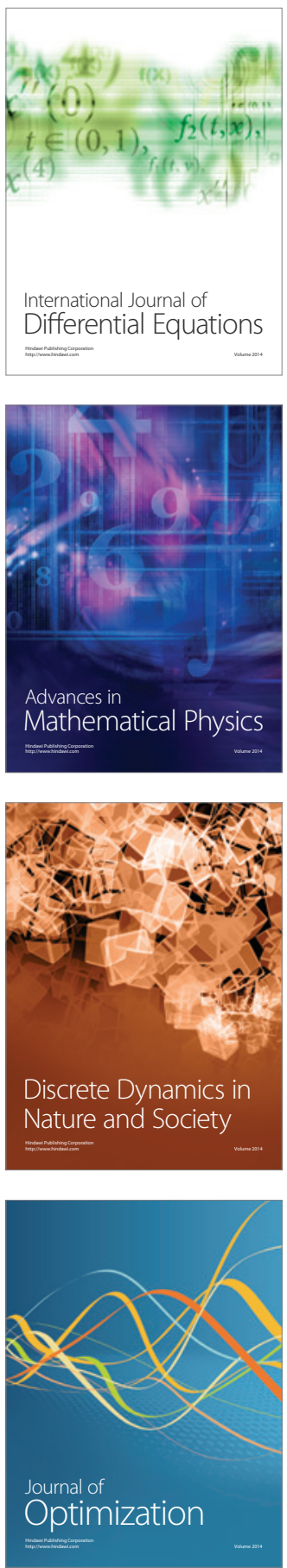